\title{
Angiotensinogen M235T polymorphism and the risk of myocardial infarction and stroke among hypertensive patients on ACE-inhibitors or $\beta$-blockers
}

Hedi Schelleman ${ }^{1,2}$, Olaf H Klungel ${ }^{2}$, Jacqueline CM Witteman ${ }^{1}$, Monique MB Breteler ${ }^{1}$, Moygan Yazdanpanah ${ }^{1}$, AH Jan Danser ${ }^{3}$, Albert Hofman ${ }^{1}$, Cornelia M van Duijn ${ }^{1}$, Anthonius de Boer ${ }^{2}$, Bruno HCh Stricker*,1

\author{
${ }^{1}$ Department of Epidemiology \& Biostatistics, Erasmus MC, Rotterdam, The Netherlands; ${ }^{2}$ Department of \\ Pharmacoepidemiology and Pharmacotherapy, Utrecht Institute for Pharmaceutical Sciences (UIPS), Utrecht University, \\ Utrecht, The Netherlands; ${ }^{3}$ Department of Pharmacology, Erasmus MC, Rotterdam, The Netherlands
}

Angiotensinogen is an essential component of the renin-angiotensin system. ACE-inhibitors and $\beta$-blockers both have a direct influence on this system. To investigate whether the association between use of ACE-inhibitors or $\beta$-blockers and the risk of myocardial infarction (MI) or stroke is modified by the T-allele of the angiotensinogen M235T polymorphism. In this study, 4097 subjects with hypertension , aged 55 years and older, were included from the Rotterdam Study, a population-based prospective cohort study in the Netherlands, from July 1, 1991 onwards. Follow-up ended at the diagnosis date of MI, stroke, death, or the end of the study period (January 1, 2002). The drug-gene interaction on the risk of MI or stroke was determined with a Cox proportional hazard model with adjustments for each drug class as time-dependent covariates. The risk of MI was increased in current use of ACE-inhibitors with the MT or TT genotype compared to ACE-inhibitors with the MM genotype (Synergy Index (SI): 4.00; 95\% CI: $1.32-12.11$ ). A significant drug-gene interaction was not found on the risk of stroke (SI: $1.83 ; 95 \% \mathrm{Cl}: 0.95-3.54)$ in ACE-inhibitor users or between current use of $\beta$-blockers and the AGT M235T polymorphism on the risk of MI or stroke. ACE-inhibitor users with at least one copy of the 235T-allele of the AGT gene might have an increased risk of $\mathrm{MI}$ and stroke.

European Journal of Human Genetics (2007) 15, 478-484. doi:10.1038/sj.ejhg.5201789; published online 14 February 2007

Keywords: pharmacogenetics; ACE-inhibitors; $\beta$-blockers; angiotensinogen; M235T polymorphism

\section{Introduction}

Hypertension is a common disorder affecting approximately $20 \%$ of the adult populations of most developed countries, ${ }^{1}$ and is a major risk factor for cardiovascular disease. In clinical trials, antihypertensive therapy has been

${ }^{*}$ Correspondence: Professor BHCh Stricker, Department of Epidemiology \& Biostatistics, Erasmus MC, PO box 1738, 3000 DR Rotterdam, The Netherlands. Tel: + 3110 4087482; Fax: + 3110 4089382;

E-mail: b.stricker@erasmusmc.nl

Received 5 September 2006; revised 9 January 2007; accepted 10 January 2007; published online 14 February 2007 associated with a $35-40 \%$ reduction in the risk of stroke and a $20-25 \%$ reduction in the risk of myocardial infarction (MI). ${ }^{2}$ Although many effective antihypertensive drugs are available, it remains difficult to predict the effect of a particular antihypertensive agent in an individual patient. Genetic variation in genes may explain differences between individuals in their response to antihypertensive drugs.

One of the genes that might influence the response to an antihypertensive drug is the angiotensinogen (AGT) gene. AGT is one of the components of the renin-angiotensin 
system, which has a central role in the regulation of blood pressure and fluid homeostasis. AGT is cleaved by renin to form angiotensin I, which is converted to the vasoactive angiotensin II by angiotensin converting enzyme (ACE). In 1992, Jeunemaitre et al. ${ }^{3}$ reported linkage of the angiotensinogen gene locus to hypertension in hypertensive sibling pairs. Subsequent screening of the angiotensinogen gene for molecular variants led to the identification of a missense mutation, resulting in the substitution of a threonine $(\mathrm{T})$ for a methionine $(\mathrm{M})$ at codon 235. Further investigations showed that the AGT 235T-allele is in linkage disequilibrium with a guanine $(G)$ to adenosine (A) transition 6 base pairs upstream of the initiation site of transcription $(-6 \mathrm{G} / \mathrm{A})$, which may result in a higher basal transcription rate of this gene. ${ }^{4}$ In general, individuals with the TT genotype have higher plasma AGT levels (10-20\%) compared to individuals with the MM genotype. ${ }^{3,5}$ Two meta-analyses reported a significant association between the M235T polymorphism and hypertension with a combined risk of 1.2 for the 235T-allele in Caucasians, ${ }^{6,7}$ however, such an association was not found for MI or stroke. ${ }^{6,8}$

Of the four mainly prescribed antihypertensive drug classes (diuretics, $\beta$-blockers, calcium channel blockers, and ACE-inhibitors), only ACE-inhibitors and $\beta$-blockers have a direct effect on the renin-angiotensin system. ACEinhibitors inhibit the conversion from angiotensin I to angiotensin II and $\beta$-blockers inhibit the release of renin. Therefore, it is plausible that the response to these antihypertensive drug classes may be modified by the M235T polymorphism of the AGT gene. Bis et $a l^{9}$ reported that the 235T-allele was associated with a stronger reduction of the risk of non-fatal stroke in users of ACEinhibitors than in users of other antihypertensive drugs, whereas there was no difference in the risk of non-fatal MI.

The objective of our study was to determine whether the risk of MI or stroke in hypertensive patients on ACEinhibitors or $\beta$-blockers is modified by the AGT M235T polymorphism.

\section{Materials and methods Setting}

The Rotterdam Study started in 1990 as a population-based prospective follow-up study. All 10275 residents of the suburb Ommoord in Rotterdam, aged 55 years or older were invited to participate. In total, 7983 (78\%) subjects gave written informed consent. The baseline measurements took place until 1993. The design of this populationbased study has been described elsewhere. ${ }^{10}$ Information was collected on age, gender, present health status and medical history, including previous MI and stroke. All reported MIs or strokes at baseline were verified with medical records. During a physical examination, blood pressure, weight, and height were measured and blood was drawn for DNA extraction. Since the start of the Rotterdam Study, follow-up examinations have been carried out every 2-3 years.

\section{Cohort and outcome definition}

Only subjects with hypertension were included in this study. Therefore, follow-up started on the day that an elevated blood pressure was measured and/or the day that a first antihypertensive drug was prescribed, whichever came first. Blood pressure data from three examination rounds were used to determine whether a person had hypertension. If a person had an elevated blood pressure ( $>160$ / $95 \mathrm{mmHg}$ or treated with antihypertensive agents) during his first examination, he/she was considered hypertensive from this point on. If a person was hypertensive during the second examination, he/she was considered hypertensive from that point on. The 'old' definition of hypertension was applied due to the start of the study in 1990.

MI and stroke cases between January 1, 1991 and July 1, 1991 were excluded, since pharmacy records were not available before January 1, 1991, and therefore their drug history was shorter than 6 months. However, cases were not excluded if they had a MI (or stroke) before January 1, 1991 and a second MI (or stroke) after July 1, 1991 (prevalent cases). The end of the study was set at January 1, 2002. Follow-up ended on the date of the first MI (or first stroke for the analysis with stroke as primary outcome), or a censoring event (end of study period, moving out of the area, or death), whichever was earlier. All collected coronary events were verified by review of hospital discharge reports and letters from medical specialists, and classified as definitive and possible MI. Two research physicians independently coded events according to the International Classification of Diseases, 10th Revision (ICD-10). ${ }^{11}$ MI was defined as ICD codes: I21. A medical expert in cardiovascular disease also reviewed all coded events for final classification.

Stroke was defined as ICD codes: K90. Stroke research physicians reviewed information on all possible strokes and transient ischemic attacks; an experienced stroke neurologist verified all diagnosis. Subarachnoid haemorrhages and retinal strokes were excluded. Ischemic strokes were diagnosed when a patient had typical symptoms and a CT and MRI that was made within 4 weeks ruled out other diagnoses or when indirect evidence (deficit limited to one limb or completely resolved within $72 \mathrm{~h}$, atrial fibrillation in the absence of anticoagulants) pointed at an ischemic nature of the stroke. Haemorrhagic stroke was diagnosed when a relevant haemorrhage was shown on CT or MRI scan, or when the subject permanently lost consciousness or died within hours after onset of focal signs. If a stroke did not match these criteria, it was classified as unspecified. ${ }^{12}$ Sub-classification into hemorrhagic and ischemic stroke was based on neuroimaging, which was available for $64 \%$ of all cases. 


\section{Exposure definition}

Pharmacy records were available for approximately $99 \%$ of the cohort as of January 1, 1991. These records include the name of the drug, the day of dispensing, the dosage form, the number of units dispensed, the prescribed daily dose, and the Anatomical Therapeutic Chemical code of the drug. ${ }^{13}$ The exposure of interest included ACE-inhibitors and $\beta$-blockers, because of their direct effect on the reninangiotensin system.

When an MI or stroke occurred, the date was defined as the event date and the cumulative duration of use for current and past exposure of all antihypertensive drug classes on that date was calculated for each participants. Hereto, we first calculated each prescription length by dividing the number of dispensed tablets or capsules by the prescribed daily number. Each refill at the pharmacy, which occurred within 7 days after last intake from the previous prescription was considered as a continuous drug episode. Current, past, and never use were defined as mutually exclusive categories. When the event fell within a usage period, the patient was considered as currently exposed, and the cumulative number of days of current use was calculated. Similarly for those who were not current user, but had used a representative of the drug group in the past the number of days since last intake was calculated. Those who had not used the drug during the follow-up period were considered as non-user. For drug dose-effect associations, we used the defined daily dosages (DDD), which consist of the recommended daily dose for the indication hypertension in an adult.

\section{Genotyping}

Genomic DNA was extracted from whole blood samples using standard methods, described previously. ${ }^{14}$ Samples were genotyped with TaqMan allelic discrimination Assays-By-Design (Applied Biosystems, Foster City, CA, USA). Forward and reverse primer (rs699) (antisense strand) sequences were $5^{\prime}$-AGG TTT GCC TTA CCT TGG AAG TG-3' and 5'-GCT GTG ACA GGA TGG AAG ACT-3' and the minor groove binding probes were $5^{\prime}$-CTG GCT CCC ATC AGG-3' (VIC) and 5'-CTG GCT CCC GTC AGG-3' (FAM). The assays utilized $5 \mathrm{ng}$ of genomic DNA and $2 \mu \mathrm{l}$ reaction volumes. The amplification and extension protocol was as follows: an initial activation step of $10 \mathrm{~min}$ at $95^{\circ}$ preceded 40 cycles of denaturation at $95^{\circ}$ for $15 \mathrm{~s}$ and annealing and extension at $50^{\circ}$ for $60 \mathrm{~s}$. Allele-specific fluorescence was then analyzed on an ABI Prism 7900HT Sequence Detection System with SDS v 2.1 (Applied Biosystems, Foster City, CA, USA).

\section{Potential confounders}

For the analysis with MI as an outcome, we considered age, gender, systolic/diastolic blood pressure, body mass index (BMI), current and past smoking, cholesterol level (total cholesterol/high-density cholesterol) at baseline as poten- tial confounders. Adjustments for history of stroke, history of MI, history of percutaneous transluminal coronary angioplasty, history of coronary artery bypass grafting, statin use, coumarin use, ASA use, NSAID use, nitrate use, use of antidiabetic medication, history of angina, past and current use of other antihypertensive drugs, and the defined daily dose were made as time-dependent covariate. We adjusted for the combined use of other antihypertensive drug classes by adding each antihypertensive drug class separately in the model for past and current users. History of angina was defined as the use of two or more prescriptions of nitrate.

For the analysis with stroke as an endpoint, we considered the same potential confounders, but combined history of MI, history of percutaneous transluminal coronary angioplasty, and history of coronary artery bypass grafting in one variable (coronary heart disease).

\section{Statistical analyses}

The outcomes for MI and stroke were analysed separately because of their different aetiology. Both events were evaluated using a Cox proportional hazard model with time-varying exposure for each antihypertensive drug class separately. We created non-cumulative time-dependent categorical variables (yes/no) for current and past use of antihypertensive drugs and follow-up time was the timeaxis of the model. Non-use of ACE-inhibitors (in the analysis of the association between ACE-inhibitors and risk of MI or stroke) and non-use of $\beta$-blockers (in the analysis of the association between $\beta$-blockers and risk of MI or stroke) served as a reference. The associations were expressed as hazard ratios (HR) with $95 \%$ confidence limits (CI). We adjusted for all potential confounders, which caused a change in the point estimate of $10 \%$ or more.

We calculated the Synergy Index (SI), which is the ratio of the HR in susceptibles to the non-susceptibles. To investigate for example the interaction between the 235T-allele and ACE-inhibitors (or $\beta$-blocker) one dummy variable was added to the model: T-allele $(0 / 1) \times$ ACEinhibitors (or $\beta$-blocker) (0/1). A SI of one means that the HR in the two subgroups are the same and that there is no interaction on a multiplicative scale. A SI greater than one means that the joint effect of gene and drug is larger than expected from their product of their individual effect. ${ }^{15}$ All analysis were performed using SAS version 9.1 (SAS Institute, Cary, NC, USA).

\section{Results}

In total, 4097 subjects with hypertension were identified. Of these 4097 persons, 1642 persons were treated with ACE-inhibitors at any time, 2387 with $\beta$-blockers, and 2561 with other antihypertensive drugs. A subject may have contributed to one of more categories of antihypertensive drug classes during follow-up. 
In total, $98 \%$ of the samples were successfully genotyped with a concordance rate of $99 \%$. In our cohort, $35.7 \%$ of the subjects had the MM genotype and $64.3 \%$ the MT or TT genotype, respectively. Table 1 shows the baseline characteristics of the 4097 subjects stratified by AGT genotypes.

\section{Myocardial infraction}

In total, 197 subjects experienced an MI. Forty-two subjects had a MI while they were treated with ACE-inhibitors, of whom six had the MM genotype and 36 had the MT or TT genotype. In total, 17 subjects with the MM genotype and

Table 1 Baseline characteristics stratified by AGT genotype

\begin{tabular}{lcc}
\hline Characteristics & $\begin{array}{c}\text { MM } \\
(\mathrm{N}=1461)\end{array}$ & $\begin{array}{c}\text { T-allele } \\
(\mathrm{N}=2636)\end{array}$ \\
\hline Gender, female & $901(61.7 \%)$ & $1575(59.7 \%)$ \\
Age (years) & $70.6 \pm 8.9$ & $70.6 \pm 8.9$ \\
Stroke at baseline (yes) & $50(3.4 \%)$ & $103(3.9 \%)$ \\
MI at baseline (yes) & $226(15.5 \%)$ & $406(15.4 \%)$ \\
Diabetes (yes) & $171(12.5 \%)$ & $314(12.7 \%)$ \\
SBP (mmHg) & $142.8 \pm 22.0$ & $144.0 \pm 22.6$ \\
DBP (mmHg) & $74.9 \pm 11.9$ & $75.3 \pm 12.1$ \\
BMI (m/kg') & $27.0 \pm 3.9$ & $26.8 \pm 3.7$ \\
Total cholesterol/high & $5.3 \pm 1.7$ & $5.3 \pm 1.6$ \\
density cholesterol (mmol/l) & & \\
& & \\
Smoking & & \\
Current (yes) & $280(19.7 \%)$ & $531(20.7 \%)$ \\
Past (yes) & $609(42.8 \%)$ & $733(42.3 \%)$ \\
Use of ACE-inhibitors & $126(8.6 \%)$ & $231(8.7 \%)$ \\
Use of $\beta$-blockers & $319(21.8 \%)$ & $573(21.7 \%)$ \\
Use of $\alpha$-blocker & $49(3.4 \%)$ & $95(3.6 \%)$ \\
Use of low-ceiling diuretic & $267(18.3 \%)$ & $440(16.7 \%)$ \\
Use of high-ceiling diuretic & $89(6.1 \%)$ & $192(7.3 \%)$ \\
Use of calcium channel blocker & $144(9.8 \%)$ & $236(9.0 \%)$ \\
Use of statins & $45(3.1 \%)$ & $69(2.6 \%)$ \\
Use of coumarins & $65(4.4 \%)$ & $136(5.2 \%)$ \\
Use of NSAID & $126(8.6 \%)$ & $243(9.2 \%)$ \\
Use of ASA/salicylate & $213(14.6 \%)$ & $374(14.2 \%)$ \\
\hline
\end{tabular}

58 subjects with at least one copy of the T-allele had an MI when they were treated with $\beta$-blockers (Table 2).

In order to investigate the possible gene-drug interaction between ACE-inhibitors or $\beta$-blockers users and the AGT M235T polymorphism on the risk of MI, cases were grouped by current use and genotype group (see Tables 2 and 3). Subjects with the MM genotype who were currently treated with ACE-inhibitors had a non-significantly reduced risk of MI compared to subjects with the MM genotype who never used ACE-inhibitors ( $\mathrm{HR}=0.71 ; 95 \%$ CI: $0.23-2.21)$. In contrast, subjects with the MT or TT genotype who were currently treated with ACE-inhibitors had a significantly increased risk of MI compared to subjects with the MM genotype who never used ACEinhibitors ( $\mathrm{HR}=2.73 ; 95 \% \mathrm{CI}$ : $1.42-5.23$ ). Among subjects who never used ACE-inhibitors, the MT or TT genotype was not associated with the risk of MI $(\mathrm{HR}=0.99 ; 95 \% \mathrm{CI}$ : $0.67-1.45)$. The estimate for the risk of MI in subjects with at least one copy of the T-allele $(\mathrm{HR}=2.73)$ who were currently treated with ACE-inhibitors was higher than expected from the joint effect of the MT or TT

Table 3 Gene-drug interaction between ACE-inhibitors and $\beta$-blockers use and AGT M235T polymorphism on the risk of $\mathrm{Ml}$

\begin{tabular}{lll}
\hline $\begin{array}{l}\text { AGT M235T } \\
\text { genotype }\end{array}$ & $\begin{array}{l}\text { Synergy index } \\
(\mathrm{MT} / \mathrm{TT} \text { versus MM) } \\
(95 \% \mathrm{Cl})^{\mathrm{a}}\end{array}$ & $\begin{array}{l}\text { Synergy index } \\
(\mathrm{MT} / \mathrm{TT} \text { versus MM) } \\
(95 \% \mathrm{Cl})^{\mathrm{b}}\end{array}$ \\
\hline $\begin{array}{l}\text { ACE-inhibitors } \\
\mathrm{MT} / \mathrm{TT} \text { versus MM }\end{array}$ & $3.28(1.28-8.45)$ & $4.00(1.32-12.11)$ \\
$\begin{array}{l}\beta \text {-blockers } \\
\text { MT/TT versus MM }\end{array}$ & $1.37(0.64-2.91)$ & $1.30(0.60-2.83)$ \\
\hline
\end{tabular}

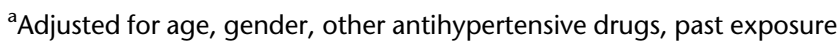
to antihypertensive drugs, and defined daily dose

${ }^{\mathrm{b}}$ Adjusted for age, gender, other antihypertensive drugs, past exposure to antihypertensive drugs, defined daily dose, BMI, cholesterol level, statin use, and history of PTCA, CABG, and MI.

Table 2 Association of ACE-inhibitors and $\beta$-blockers use and AGT M235T polymorphism with myocardial infraction (MI) risk

\begin{tabular}{llcrr}
\hline AGT M235T genotype & Type of use & MI (N) & $H R(95 \% C l)^{\mathrm{a}}$ & $H R(95 \% \mathrm{Cl})^{\mathrm{b}}$ \\
\hline ACE-inhibitors & & & & 1 (reference) \\
MM & No & 44 & $1.06(0.39-2.85)$ & $0.71(0.23-2.21)$ \\
MM & Current & 6 & $0.95(0.66-1.39)$ & $0.99(0.67-1.45)$ \\
MT/TT & No & 74 & $3.25(1.70-6.19)$ & $2.73(1.42-5.23)$ \\
MT/TT & Current & 36 & & 1 (reference) \\
B-Blockers & & & 1 (reference) & $1.29(0.59-2.86)$ \\
MM & No & 20 & 1.69 (0.79-3.61) & $1.44(0.84-2.48)$ \\
MM & Current & 17 & $1.36(0.81-2.30)$ & $2.42(1.23-4.76)$ \\
MT/TT & No & 49 & $3.16(1.66-6.01)$ & \\
MT/TT & Current & 58 &
\end{tabular}

${ }^{a}$ Adjusted for age, gender, other antihypertensive drugs, past exposure to antihypertensive drugs, and defined daily dose.

${ }^{b}$ Adjusted for age, gender, other antihypertensive drugs, past exposure to antihypertensive drugs, defined daily dose, BMI, cholesterol level, statin use, and history of PTCA, CABG, and MI. 
genotype and ACE-inhibitors on a multiplicative scale $(0.99 \times 0.71=0.70)$. This interaction between current use of ACE-inhibitors and the AGT M235T polymorphism was statistically significant ( $\mathrm{SI}=4.00 ; 95 \% \mathrm{CI}$ : $1.32-12.11)$. There did not seem to be a dose-response effect (MT versus MM genotype $\mathrm{SI}=3.89 ; 95 \% \mathrm{CI}: 1.27-11.90$ and TT versus MM genotype $\mathrm{SI}=3.75$; $95 \% \mathrm{CI}: 1.04-13.58$ ).

$\beta$-Blocker users who had the MM genotype had a nonsignificantly increased risk of MI compared to subjects with the MM genotype who never used $\beta$-blockers $(\mathrm{HR}=1.29$; 95\% CI: 0.59-2.86). Compared to subjects with the MM genotype who had never used $\beta$-blockers, $\beta$-blocker users with the MT or TT genotype had a significantly increased risk of $\mathrm{MI}(\mathrm{HR}=2.42 ; 95 \% \mathrm{CI}: 1.23-4.76)$. The interaction between current use of $\beta$-blockers and the AGT M235T polymorphism was not significant ( $\mathrm{SI}=1.30$; 95\% CI: 0.60-2.83). Additional analyses in which adjustments were made for systolic blood pressure level, diastolic blood pressure level, history of angina, use of ASA, use of coumarins, use of NSAID's, use of antidiabetic medication, history of stroke, and smoking, yielded similar results and were therefore not shown.

In total, 72 subjects with a MI had a prior MI before 1990. After exclusion of all subjects with a history of MI $(n=632)$ the (adjusted) interaction between AGT M235T polymorphism and current use of ACE-inhibitors was borderline significant ( $\mathrm{SI}=4.62$; 95\% CI: 1.00-21.36). No druggene interaction was found between the AGT M235T polymorphism and use of $\beta$-blockers after this exclusion (SI $=1.19 ; 95 \%$ CI: 0.47-3.02).

When the analysis was repeated with other antihypertensive drug classes (i.e. low-ceiling diuretics or calcium channel blockers) there was no significant drug-gene interaction with any of these antihypertensive drug classes.

\section{Stroke}

In total, 349 subjects experienced a stroke during followup. Of the 365 events, 189 (85\%) were classified as ischemic and $33(15 \%)$ as hemorrhagic. Sixty-three subjects had a stroke when they were treated with an ACE-inhibitor, of whom 15 had the MM genotype and 48 had at least one copy of the T-allele. During treatment with a $\beta$-blocker, 32 subjects with the MM genotype and 71 subjects with had at least one copy of the T-allele had a stroke.

To investigate the possible interaction between ACE-inhibitors or $\beta$-blockers users and the AGT M235T polymorphism on the risk of stroke, participants were grouped by current use and genotype group (see Tables 4 and 5). The drug-gene interaction between current use of ACE-inhibitors and the AGT M235T polymorphism on the risk of MI was increased but this increase was not statistically significant $(\mathrm{SI}=1.83 ; 95 \%$ CI: 0.95-3.54). There was no allele dose-response effect in ACE-inhibitor users (MT versus $\mathrm{MM}$ genotype $\mathrm{SI}=1.88$; 95\% CI: $0.96-3.69$ and TT versus $\mathrm{MM}$ genotype $\mathrm{SI}=1.93$; 95\% CI: 0.80-4.66).

The interaction between current use of $\beta$-blockers and the AGT M235T polymorphism on the risk of stroke was non-significant ( $\mathrm{SI}=1.39 ; 95 \% \mathrm{CI}$ : 0.81-2.39). Additional

Table 5 Gene-drug interaction between ACE-inhibitors and $\beta$-blockers use and AGT M235T polymorphism on the risk of stroke

\begin{tabular}{lll}
\hline $\begin{array}{l}\text { AGT M235T } \\
\text { genotype }\end{array}$ & $\begin{array}{l}\text { Synergy index } \\
(\mathrm{MTT/TT} \text { versus MM) } \\
(95 \% \mathrm{Cl})^{\mathrm{a}}\end{array}$ & $\begin{array}{l}\text { Synergy index } \\
(\mathrm{MTT/TT} \text { versus MM) } \\
(95 \% \mathrm{Cl})^{\mathrm{b}}\end{array}$ \\
\hline $\begin{array}{l}\text { ACE-inhibitors } \\
\mathrm{MT} / \mathrm{TT} \text { versus MM }\end{array}$ & $1.93(1.02-3.65)$ & $1.83(0.95-3.54)$ \\
$\begin{array}{l}\beta \text {-Blockers } \\
\text { MT/TT versus MM }\end{array}$ & $1.41(0.84-2.38)$ & $1.39(0.81-2.39)$ \\
\hline
\end{tabular}

${ }^{a}$ Adjusted for age, gender, other antihypertensive drugs, past exposure to antihypertensive drugs, and defined daily dose.

${ }^{\mathrm{b}}$ Adjusted for age, gender, other antihypertensive drugs, past exposure to antihypertensive drugs, defined daily dose, systolic blood pressure level, diabetes mellitus, use of statins, use of ASA, and history of coronary heart disease, stroke, and angina.

Table 4 Association of ACE-inhibitors and $\beta$-blockers use and AGT M235T polymorphism with stroke risk

\begin{tabular}{|c|c|c|c|c|}
\hline AGT M235T genotype & Type of use & Stroke $(N)$ & $H R(95 \% C I)^{\mathrm{a}}$ & $H R(95 \% C I)^{\mathrm{b}}$ \\
\hline \multicolumn{5}{|l|}{ ACE-inhibitors } \\
\hline $\mathrm{MM}$ & No & 95 & 1 (reference) & 1 (reference) \\
\hline MM & Current & 15 & $0.64(0.33-1.23)$ & $0.58(0.29-1.14)$ \\
\hline $\mathrm{MT} / \mathrm{TT}$ & No & 141 & $0.84(0.65-1.09)$ & $0.84(0.64-1.10)$ \\
\hline $\mathrm{MT} / \mathrm{TT}$ & Current & 48 & $1.04(0.63-1.71)$ & $0.89(0.53-1.48)$ \\
\hline \multicolumn{5}{|l|}{$\beta$-Blockers } \\
\hline $\mathrm{MM}$ & No & 66 & 1 (reference) & 1 (reference) \\
\hline $\mathrm{MM}$ & Current & 32 & $0.68(0.40-1.14)$ & $0.73(0.43-1.23)$ \\
\hline $\mathrm{MT} / \mathrm{TT}$ & No & 104 & $0.85(0.63-1.16)$ & $0.85(0.62-1.21)$ \\
\hline $\mathrm{MT} / \mathrm{TT}$ & Current & 71 & $0.82(0.51-1.30)$ & $0.86(0.54-1.39)$ \\
\hline
\end{tabular}

${ }^{a}$ Adjusted for age, gender, other antihypertensive drugs, past exposure to antihypertensive drugs, and defined daily dose.

${ }^{b}$ Adjusted for age, gender, other antihypertensive drugs, past exposure to antihypertensive drugs, defined daily dose, systolic blood pressure level, diabetes mellitus, use of statins, use of ASA, and history of coronary heart disease, stroke, and angina. 
analyses in which we adjusted for diastolic blood pressure level, serum cholesterol level, BMI, use of coumarins, use of NSAID's, and smoking, yielded similar results and were therefore not shown.

In total, 27 subjects with a stroke had a prior stroke before 1990. After exclusion of all subjects with a history of stroke $(n=153)$ the (adjusted) interaction between AGT M235T polymorphism and current use of ACE-inhibitors was significant $(\mathrm{SI}=2.14 ; 95 \% \mathrm{CI}$ : $1.06-4.33)$. No druggene interaction was found between $\beta$-blocker use and the AGT M235T polymorphism after this exclusion ( $\mathrm{SI}=1.59$; 95\% CI: 0.90-2.78). When we included only ischemic strokes, the interaction was significant for ACE-inhibitors $(\mathrm{SI}=3.52 ; 95 \% \mathrm{CI}$ : $1.27-9.80)$, but not for $\beta$-blockers $(\mathrm{SI}=1.30 ; 95 \% \mathrm{CI}: 0.47-3.02)$.

When the analysis was repeated with other antihypertensive drug classes (i.e. low-ceiling diuretics or calcium channel blockers) there was no significant drug-gene interaction with current use.

\section{Discussion}

In this study, a synergistic interaction between the AGT M235T polymorphism and current use of ACE-inhibitors on the risk of MI was found. The joint effect in T-allele carriers was approximately four times greater than expected based on the product of their individual effects. In TT homozygotes, similar results were observed. Overall no significant interaction was found on the risk of stroke, although, the direction of the synergistic effect was similar as to that on the risk of MI. However, after exclusion of all subjects with a history of stroke, there was a statistically significant interaction between the AGT M235T polymorphism and ACE-inhibitors, especially after further restriction to cases of ischemic stroke. No interaction was found between the use of $\beta$-blockers and the M235T polymorphism.

Bis et $a l^{9}$ reported that users of ACE-inhibitors carrying one copy of the T-allele might have a reduced risk of nonfatal stroke compared to users of other antihypertensive drugs and an non-significantly increased risk for non-fatal MI. In our study we found the opposite result for stroke, that is, the risk of stroke in $\beta$-blockers was higher in subjects carrying a copy of the 235T-allele, albeit not significant. In addition, in our study the MT and TT genotype were associated with an increased risk of MI in ACE-inhibitors users. In a non-randomized trial, ACEinhibitor users with the MT or TT genotype had a greater BP reduction than those with the MM genotype. ${ }^{16}$ However, this could not be replicated in a smaller study. ${ }^{17}$ With regard to $\beta$-blockers, no drug-gene interaction on $\mathrm{BP}$ was found. ${ }^{17,18}$ The contribution of these findings on blood pressure to cardiovascular risk remains uncertain. For example, no studies have been published about an interaction between the M235T polymorphism and antihypertensive drugs on the risk of atherosclerosis, which is an important risk factor for MI and stroke besides blood pressure. There are some differences between our study and Bis et $a l^{9}$ which might explain the differences in results. For instance, we included fatal and non-fatal cases of MI and stroke, adjusted for past use, and compared the use of ACEinhibitor or $\beta$-blockers versus non-users instead of users of other antihypertensive drugs. We cannot determine which of the two studies is more likely to be correct, because it is difficult to predict with accuracy which allele would be associated with an increased risk of stroke or MI during treatment with an ACE-inhibitor or $\beta$-blocker. For example, although, plasma AGT levels are $10-20 \%$ higher in TT homozygotes than in MM homozygotes ${ }^{3,5}$ this did not result in higher angiotensin II levels due to compensation in renin levels. ${ }^{19,20}$

The main limitation of our study is the relatively small number of events. Although, our analyses consistently showed that current use of ACE-inhibitors among subjects with the T-allele was associated with an increased risk of MI and stroke, the results should still be interpreted with caution. A small number of events was also the main limitation of the study of Bis et al. ${ }^{9}$ Therefore, these results need to be replicated in other studies before definitive conclusions can be made. In addition, due to limited sample size we were unable to test for dose-response and duration-response relationships. Furthermore, we compared the risk of MI or stroke in antihypertensive drug users versus non-users and therefore confounding by indication could have biased our results if non-users had a better prognosis at baseline. As a physician was free to choose whether a patient received antihypertensive drug treatment or not and the type of antihypertensive drug, specific patients characteristics may have influenced this decision. However, the interaction between $\beta$-blockers or ACE-inhibitors and the AGT M235T polymorphism is probably not influenced by this bias, as the users of the same antihypertensive drug class will have most likely the same characteristics and the prescriber is unaware of a subject's genotype. Furthermore, only one SNP in the AGT gene was investigated. Although the M235T polymorphism is linked with plasma AGT levels and hypertension, it remains controversial in relation to MI and stroke. Since more genes are involved in the renin-angiotensin system and these might have compensated the increased risk of ACE-inhibitors in subjects with the T-allele for example by lowering the production of renin. One explanation of our finding might be that the feedback mechanisms that normally compensate a rise in the angiotensinogen (e.g. a decrease in renin) are dysfunctional in T-allele carriers. This might be of particular importance during treatment with ACE-inhibitors, since such a treatment is known to be accompanied by a compensatory rise in renin. Future studies, addressing plasma renin levels during ACE- 
inhibitor treatment in T-allele carriers and controls, should evaluate this possibility.

In conclusion, our results indicate that the T-allele might be associated with an increased risk of MI and stroke in users of ACE-inhibitors. However, due to our limited sample size our findings require replication.

\section{Acknowledgements}

The Netherlands Heart Foundation financially supported this study, Grant number: 2001.064 The Rotterdam Study is supported by the Erasmus Medical Center and Erasmus University Rotterdam, the Netherlands Organization for Scientific Research (NWO), the Netherlands Organization for Health Research and Development (ZonMw), the Research Institute for Diseases in the Elderly (RIDE), the Ministry of Education, Culture and Science, the Ministry of Health, Welfare and Sports, the European Commission (DG XII), the Municipality of Rotterdam, and the Centre for Medical Systems Biology (CMSB). The contributions of the general practitioners and pharmacists of the Ommoord district to the Rotterdam Study are greatly acknowledged.

\section{References}

1 Brown MJ, Haydock S: Pathoaetiology, epidemiology and diagnosis of hypertension. Drugs 2000; 59 (Suppl 2): 1-12.

2 Neal B, MacMahon S, Chapman N: Effects of ACE inhibitors, calcium antagonists, and other blood-pressure-lowering drugs: results of prospectively designed overviews of randomised trials. Blood Pressure Lowering Treatment Trialists' Collaboration. Lancet 2000; 356: 1955-1964.

3 Jeunemaitre X, Soubrier F, Kotelevtsev YV et al: Molecular basis of human hypertension: role of angiotensinogen. Cell 1992; 71: $169-180$.

4 Inoue I, Nakajima T, Williams CS et al: A nucleotide substitution in the promoter of human angiotensinogen is associated with essential hypertension and affects basal transcription in vitro. J Clin Invest 1997; 99: 1786-1797.

5 Schunkert H, Hense HW, Gimenez-Roqueplo AP et al: The angiotensinogen T235 variant and the use of antihypertensive drugs in a population-based cohort. Hypertension 1997; 29: 628633.

6 Sethi AA, Nordestgaard BG, Tybjaerg-Hansen A: Angiotensinogen gene polymorphism, plasma angiotensinogen, and risk of hypertension and ischemic heart disease: a meta-analysis. Arterioscler Thromb Vasc Biol 2003; 23: 1269-1275.

7 Kunz R, Kreutz R, Beige J, Distler A, Sharma AM: Association between the angiotensinogen 235T-variant and essential hyper- tension in whites: a systematic review and methodological appraisal. Hypertension 1997; 30: 1331-1337.

8 Sethi AA, Tybjaerg-Hansen A, Gronholdt ML, Steffensen R, Schnohr P, Nordestgaard BG: Angiotensinogen mutations and risk for ischemic heart disease, myocardial infarction, and ischemic cerebrovascular disease. Six case-control studies from the Copenhagen City Heart Study. Ann Intern Med 2001; 134: 941-954.

9 Bis JC, Smith NL, Psaty BM et al: Angiotensinogen Met235Thr polymorphism, angiotensin-converting enzyme inhibitor therapy, and the risk of nonfatal stroke or myocardial infarction in hypertensive patients. Am J Hypertens 2003; 16: 1011-1017.

10 Hofman A, Grobbee DE, de Jong PT, van den Ouweland FA: Determinants of disease and disability in the elderly: the Rotterdam Elderly Study. Eur J Epidemiol 1991; 7: 403-422.

11 World Health organization: International Classification of Diseases, 10th Revision (ICD-10). Geneva: WHO Switzerland: World Health Organization, 1992.

12 Hollander M, Bots ML, Del Sol AI et al: Carotid plaques increase the risk of stroke and subtypes of cerebral infarction in asymptomatic elderly: the Rotterdam study. Circulation 2002; 105: 2872-2877.

13 Anonymous: Guidelines for ATC classification and DDD assignment, 3rd edn, Oslo: WHO collaberating Centre for drug statistics Methodology, 2000.

14 van Duijn CM, de Knijff P, Cruts M et al: Apolipoprotein E4 allele in a population-based study of early-onset Alzheimer's disease. Nat Genet 1994; 7: 74-78.

15 Khoury MJ, Flanders WD: Nontraditional epidemiological approaches in the analysis of gene-environment interaction: case-control studies with no controls!. Am J Epidemiol 1996; 144: $207-213$.

16 Hingorani AD, Jia H, Stevens PA, Hopper R, Dickerson JE, Brown MJ: Renin-angiotensin system gene polymorphisms influence blood pressure and the response to angiotensin converting enzyme inhibition. J Hypertens 1995; 13: 1602-1609.

17 Dudley C, Keavney B, Casadei B, Conway J, Bird R, Ratcliffe P: Prediction of patient responses to antihypertensive drugs using genetic polymorphisms: investigation of renin-angiotensin system genes. J Hypertens 1996; 14: 259-262.

18 Kurland L, Melhus H, Karlsson J et al: Angiotensin converting enzyme gene polymorphism predicts blood pressure response to angiotensin II receptor type 1 antagonist treatment in hypertensive patients. J Hypertens 2001; 19: 1783-1787.

19 Danser AH, Derkx FH, Hense HW, Jeunemaitre X, Riegger GA, Schunkert H: Angiotensinogen (M235T) and angiotensin-converting enzyme (I/D) polymorphisms in association with plasma renin and prorenin levels. J Hypertens 1998; 16: 1879-1883.

20 Hopkins PN, Lifton RP, Hollenberg NK et al: Blunted renal vascular response to angiotensin II is associated with a common variant of the angiotensinogen gene and obesity. $J$ Hypertens 1996; 14: 199-207. 